\title{
Role of C Reactive Protein in Acute Appendicitis: A Cross-Sectional Study
}

\section{Lamture Yeshwant Ramrao1, Varsha Gajbhiye², Vikram Panjabrao Vaidya ${ }^{3}$, Md. Jawed Akther ${ }^{1}$, Mangesh Padmawar ${ }^{4}$}

\begin{abstract}
'Professor, Department of Surgery, J.N. Medical College, Datta Meghe Institute of Medical Sciences, Wardha, MS, India; ${ }^{2}$ Associate Professor, Department of Pharmacology, Datta Meghe Medical College, Nagpur, MS, India; ${ }^{3}$ Assistant Professor, Department of Surgery, ].N. Medical College, Datta Meghe Institute of Medical Sciences, Wardha, MS, India; ${ }^{A}$ Associate Professor, Department of Surgery, Datta Meghe Medical College, Nagpur, MS, India.
\end{abstract}

\section{ABSTRACT}

Introduction: The diagnosis of acute appendicitis is still difficult and probably the commonest difficulty in the emergency surgical department. Raised levels of inflammatory markers like $\mathrm{C}$ reactive protein (CRP) in a clinically suspected case of acute appendicitis suggest early exploration. Appendicitis is an inflammatory process in the appendix. There is no confirmatory tool to detect this inflammation and its sequelae. C-reactive protein is synthesized in the liver in the response of any sort of insult to the body including inflammation lead to the rise of a level. Diagnostic accuracy of CRP is a more than ESR and leucocyte count in detecting inflammatory processes. Hence this study was conducted to know the significance of CRP to detect acute appendicitis.

Material and Method: This Prospective cross-sectional study was inclusive of 100 Patients of acute appendicitis with no age bar. Subjected for appendicectomy and resected specimen for histopathological examination, CRP was measured before surgery.

Result: C-reactive protein was raised in 62 out of 100 patients. The sensitivity and specificity of was $81.28 \%$ and $92.86 \%$ with negative and positive predictive values were $26.26 \%$ and $99.37 \%$ respectively. Higher levels of CRP more than $15 /$ dl were suggestive of complicated appendicitis like perforation and gangrene.

Conclusion: CRP is a valuable investigation in acute appendicitis. CRP is a valuable tool to rule out acute appendicitis and complications but its value to detect or diagnose acute appendicitis is low.

Key Words: Inflammation, Gangrenous appendicitis, Perforation, C reactive protein, Erythrocyte sedimentation rate

\section{INTRODUCTION}

The typical presentation of acute appendicitis was firsttime described by Reginald Haber Fitz in the late seventeenth century. From that time, it was the most frequent presentation of emergency surgery patients. Around $6 \%$ of the peoples will be affected by acute appendicitis during their lifetime; hence, very strong efforts are already delivered for correct identification of appendicitis and its treatment. Due to this, decreases the death rate and morbidity significantly even with complications. The detection of appendicitis sometimes a hard task, hence doubtful atypical patients need admission and repeated examinations. This can lead to delay in diagnosis ultimately affect to raise the possibility of complications ${ }^{1}$.
A negative appendectomy means a normal histopathology specimen. Various tools have been suggested to help in atypical cases to lower the negative appendectomy rates (NAR). Many scoring systems have been used to detect early acute appendicitis and its surgery. These scores are based on clinical findings and blood investigations. In all patients, but, a final diagnosis is only by histopathological examination of the appendix. Without removal, the diagnosis of acute appendicitis is not correct in most cases, and being bad in females than in males. ${ }^{2}$.

C-reactive protein (CRP) is quite a routine laboratory test performed, to assess and identify the inflammatory process. But, the functions of CRP in normal health is not distinctly understood but increases its level by many folds during the

\section{Corresponding Author:}

Dr. Md. Jawed Akther, Professor, Department of Surgery, J.N. Medical College, Datta Meghe Institute of Medical Sciences, Wardha, MS, India; Email: dr.mdjawedakther1973@gmail.com; yash18671@gmail.com

ISSN: 2231-2196 (Print)

Received: 16.07 .2020
ISSN: 0975-5241 (Online)

Revised: 26.08 .2020
Accepted: 30.09 .2020

Published: 27.10 .2020 
event of insult to the body. Hence it is used as a tool to identify inflammatory disease process $^{3}$.

Increased levels of $\mathrm{C}$ reactive protein (CRP) in a clinically suspected case of acute appendicitis suggest early exploration. $\mathrm{C}$ - reactive protein is synthesized in the liver in the response of any sort of insult to the body including inflammation lead to the rise of the level. Diagnostic accuracy of CRP is a more than ESR and leucocyte count in detecting inflammatory processes. Hence investigation like an estimation of CRP in suspected appendicitis may help to improve the diagnostic ability of clinician ${ }^{4}$.

Many studies have proved the role of CRP in increasing the accuracy of the diagnosis of acute appendicitis ${ }^{5,6}$. It is a more specific and a good parameter of inflammation than ESR and white cell count. The CRP levels rise faster than that of ESR and decrease quickly when the inflammation subsides. A false-negative test can get usually early in infective episodes

Birchley D in his study results stated that in suspected cases of acute appendicitis posted for surgery based on clinical examination total leucocyte count and granulocyte count differentiate acute appendicitis from normal appendices but do not suggest the presence of appendicular perforation or abscess. C-reactive protein not able to differentiate appendicitis from normal, but suggests the presence of appendicular abscess at higher levels only. A study conducted by Lateef et al.8 suggested that it is possible to get inflamed appendix even inflammatory markers not raised.

Looking at this confusing scenario, we undertake this study to assess the significance of this inflammatory marker, CRP in a diagnosis of acute appendicitis.

\section{METHODOLOGY}

The present study was undertaken in the department of surgery, Jawaharlal Nehru Medical College, Wardha in collaboration with Datta Meghe Medical College Hingana, Nagpur, Datta Meghe Institute of medical science (DMIMS), Sawangi, Meghe, Wardha, Maharashtra India. This study was a prospective interventional. The duration of this study was from September 2019 to June 2020. The numbers of patients were 100 .

Sample size- 100

Type of study (research design): Prospective cross-sectional study.

Period of study: September 2019 to June 2020

\section{Inclusion criteria}

Clinically diagnosed cases of Acute Appendicitis.

\section{Exclusion criteria}

- Pregnancy

- Appendicular lump.

- Inflammatory Disease and connective tissue disorders like inflammatory bowel diseases or sickle cell disease

- HIV positive,

- Patients on corticosteroid therapy,

- Patient of interval appendectomy

\section{Data collection tools and process, variables, definitions, analysis plan}

Informed consent was obtained before enrollment. Approval of the institutional ethical committee was sought. After careful examination and appropriate imaging and blood investigations including estimation of CRP. The diagnosis of appendicitis was confirmed by a senior consultant. An appendectomy was performed and a specimen was sent for histopathological examination.

The data analysis was done by using the SPSS 17.0 statistical software, the sensitivity, specificity, positive predictive value (PPV), negative predictive value (NPV), and diagnostic accuracy of CRP was calculated.

Ethical approval for the study was got from the Ethics committee of DMIMS University

\section{RESULT}

Around 100 subjects were included in the study, consists of 60 males and 40 from the female gender. All patients were operated by the laparoscopic appendicectomy method (figure 1).

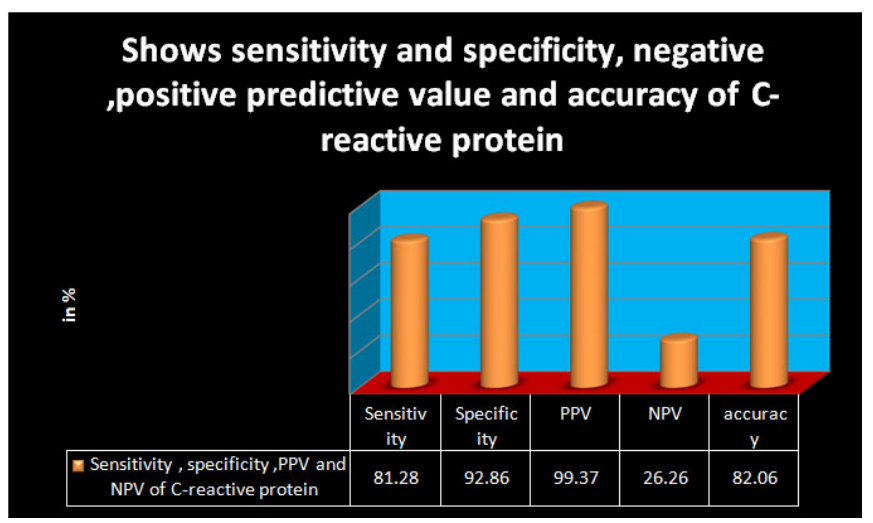

Figure 1: Shows sensitivity and specificity, negative, positive predictive value, and accuracy of C-reactive protein.

In the present study, we found the sensitivity and specificity of C-reactive protein was $81.28 \%$ and $92.86 \%$ with negative and positive predictive values were $26.26 \%$ and $99.37 \%$ respectively (Figure 2). 


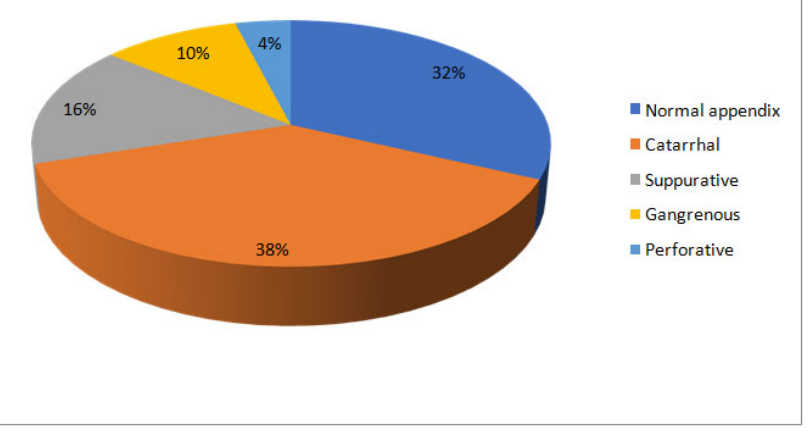

Figure 2: Pathological diagnosis as per the Histopathological Report in percentages.

The present study, figure $1 \& 2$ suggest normal appendix in 32 patients out of 100 patients, 38 patients have catarrhal appendicitis, 16, 10 and 4 patients were diagnosed suppurative, gangrenous and perforated appendicitis respectively as per of histopathological examination.

\section{DISCUSSION}

An inflammatory marker, C-reactive protein (CRP) studied in the present research. It is an important parameter to help in detecting acute appendicitis in an early course of this disease process. If it is normal, the diagnosis of appendicitis must be reconfirmed by repeated clinical examination reinforced with ultrasonography or diagnostic laparoscopy. The typical combination of three, a history, clinical examination, and CRP has a higher accuracy power to detect appendicitis in most of the patients ${ }^{9}$.

In the present study, including more males than females (3:2), the sensitivity and specificity of C-reactive protein was $81.28 \%$ and $92.86 \%$ with negative and positive predictive values were $26.26 \%$ and $99.37 \%$ respectively (see fig. 1 and 2). In the present study, CRP was positive in 62 out of 100 patients. A study conducted by Giorgio Stefanutti et al ${ }^{10}$ proves that if CRP levels are normal in a child with even high clinical suspicion of acute appendicitis, the presence of an inflamed appendix is extremely rare and re-examination with appropriate investigations are required before attempting removal of the appendix. Results of a study done by Giorgio Stefanutti et al. ${ }^{10}$ are similar to the present study.

In a present study, a high negative appendectomy rate of $32 \%$ was observed (see (figure 3 ) Wani et $\mathrm{al}^{2}$ also suggested a NAR of $20-40 \%$ has been documented and many surgeons consider a rate of $30 \%$ as inevitable. Removal of a normal appendix is an economic loss on both the patients and the hospital. Misdiagnosis and delay in surgery can lead to complications like perforation and finally peritonitis ${ }^{2}$.
The present study suggest normal appendix in 32 patients out of 100 patients, 38 patients have catarrhal appendicitis, 16,10 and 4 patients were diagnosed suppurative, gangrenous and perforated appendicitis respectively as per of histopathological examination. All complicated appendicitis were associated with higher levels of CRP more than $15 \mathrm{mg}$ /dl. Choudhary SK et al. suggested that CRP useful to correctly predict the severity of acute appendicitis. higher CRP has a high sensitivity for complications of acute appendicitis than specificity. He also suggests the cut off level at $6 \mathrm{mg} /$ $\mathrm{dL}$ requires to be used. If clinical presentations and imaging suggestive of appendicitis, a person with high CRP should undergo surgery urgently ${ }^{4}$.

Alvarado $\mathrm{A}^{6}$ in his review article depicts that a few inflammatory parameters, alone or combined, do well to help the detection of acute appendicitis in the late stages. But, not superior to the clinical examination of patients suspected of acute appendicitis. The duration of pain and other symptoms easily suggest that whether the inflammatory process is early or late. Hence clinical examination has a higher place to do a correct diagnosis.

D Birchley et al.7 suggested that: 'components of the history, clinical presentation and laboratory investigation are poor identifiers of acute appendicitis. But if combined, they provide high diagnostic ability.' Hence it is wise to say that in combination, CRP has a raised diagnostic accuracy and more effective in helping a clinical diagnosis of acute appendicitis.

Very high levels of CRP either alone or coupled with raised TLC warrants mandatory explorations. The present study recommends that CRP should be done as a routine laboratory test in the doubtful diagnosis of acute appendicitis.

\section{CONCLUSION}

In conclusion, $\mathrm{CRP}$ is an important inflammatory marker in acute appendicitis. Because it is not specific, hence it has limitations. The simultaneous presence of other inflammatory disease processes, and the effect of non-inflammatory agents, like tobacco chewing and smoking, high body mass index, and psychological stress, decreases the correctness of CRP significantly. Because of these factors, clinical correlation with imaging is required in atypical cases., Hence CRP is a valuable tool to rule out acute appendicitis and complications but its value to detect or diagnose acute appendicitis is low.

\section{ACKNOWLEDGMENT}

Authors acknowledge the immense help received from the scholars whose articles are cited and included in references to this manuscript. The authors are also grateful to authors / editors / publishers of all those articles, journals, and books 
from which the literature for this article has been reviewed and discussed.

\section{Conflict of Interest: Nil}

\section{Source of Funding: Nil}

\section{REFERENCES}

1. Ahmed $\mathrm{M}$ et al. Appraisal of the modified Alvarado score for acute appendicitis in adults. Saudi Medical Journal 2004; 25 (9): 1229-1231.

2. Wani, $\mathrm{M}$ et al. Usefulness of the Alvarado Scoring System Concerning Age, Sex and Time of Presentation, with Regression Analysis of Individual Parameters. The Internet Journal of Surgery. $2007 ;$ 11(2).

3. Chandrashekara S. C-reactive protein: An inflammatory marker with a specific role in physiology, pathology, and diagnosis. IJRCI. 2014;2(S1): SR3.
4. Khairy G. et al. acute appendicitis: Is removal of a normal appendix still existing and can we reduce its rate? Saudi J Gastroenterol 2009; 15:167-70.

5. Choudhary $\mathrm{S}$ et al. Diagnostic value of $\mathrm{C}$-reactive protein as a predictor of complicated appendicitis like perforated/gangrenous appendicitis. Int Surg J 2019; 6:1761-6.

6. Alvarado A. Inflammatory Markers in Acute Appendicitis: Are We Still Looking for the Philosopher's Stone? J Surg, 2018; 02: JSUR-1104.

7. Birchley D, Patients with clinical acute appendicitis should have pre-operative full blood count and c-reactive protein assays, Ann R Coll Surg Engl. 2006;88(1):27-32.

8. Ibrahim, $M$ et al. Ultrasonography in the Diagnosis of Clinically Equivocal Acute Appendicitis: A Prospective Study Kuwait Medical Journal 2003, 35 (3): 271-274.

9. Lateef A et al. Role of leucocyte count in the diagnosis of acute appendicitis, Gomal Journal of Medical Sciences. 2009, 7(2): 140-142.

10. Stefanutti $G$ et al. Inflammatory markers for acute appendicitis in children: Are they helpful? Journal of Paediatric Surgery. 2007; 42, 773-776. 